\title{
Influence of periodontitis and nonsurgical periodontal intervention on atherosclerosis diseases
}

\author{
Tielou Chen ${ }^{1}$, Shifeng Wang ${ }^{2}$, Guoqin Liu ${ }^{1}$, Xinhai Zhang ${ }^{1}$, Dahai Tang ${ }^{3}$, Zhifen Wu ${ }^{4}$ \\ ${ }^{1}$ Department of Periodontol, Diagnosis \& Treatment Center of Stomatological Diseases of CPLA, Hospital 411 of CPLA, Shanghai, \\ China \\ ${ }^{2}$ Naval Medical Research Institute, Shanghai, China \\ ${ }^{3}$ Department of Clinical Medical Laboratory, Hospital 411 of CPLA, Shanghai, China \\ ${ }^{4}$ Department of Periodontol, College of Stomatology, Fourth Military Medical University, Xi'an, China \\ Email: ${ }^{*}$ chentielou2010@sina.com
}

Received 30 June 2012; revised 30 July 2012; accepted 16 August 2012

\begin{abstract}
Objective: Periodontitis and atherosclerosis diseases are chronic inflammatory disorders which are highly prevalent in populations. Nonsurgical periodontal intervention belongs to the initial therapy strategy to periodontal diseases. Periodontal pathogen can enter into blood stream through the ulceration epithelial resulting in bacteraemia when periodontitis is severe. The objective is to investigate the relationship between periodontitis and atherosclerosis diseases, and the influence of nonsurgical periodontal intervention on atheroma and atherosclerosis diseases. Methods: This study reviewed and analyzed the papers which published in the world associated with periodontitis or periodontal intervention on atherosclerosis diseases. Results: Periodontitis and periodontal infectious are important risk factors for atherosclerotic diseases. Much evidence has proved the durative severe periodontitis can result in bacteraemia and systemic inflammation, elevated C-response protein in serum, gingival microcirculation changed, periodontal microorganism reproduced, and endothelial dysfunction and endocarditis. Nonsurgical periodontal intervention can remove the pathogenesis bacteria and calculus to recover periodontal health. Effective periodontal therapy can reduce bacteraemia and stop the hurt to vessels. Nonsurgical periodontal therapy may interfere periodontal bacteria, inhibit inflammation response and C-response protein, improving gingival microcirculation and vessel epithelial function to prevent atherosclerosis. Conclusion: Nonsurgical periodontal intervention can improve or decrease the rate of atherosclerotic disease by interfere the severe periodontitis. The detailed mechanism of periodontal intervention on atheroma and atherosclerotic disease is still need to be explored.
\end{abstract}

${ }^{*}$ Corresponding author.
Keywords: Atherosclerosis Diseases; Nonsurgical Periodontal Intervention; Periodontitis; Epithelial Function; C-Response Protein

\section{INTRODUCTION}

Periodontitis and atherosclerosis diseases are common chronic inflammatory disorders which are most prevalent in worldwide. Periodontal attachment loss and alveolar bone absorption are the main clinical symptom. Periodontal supportive tissue inflammatory and destructive induced by dental plaque. Nonsurgical periodontal intervention, which are initial therapeutic strategy to periodontal diseases, have the therapeutic effects to periodontitis and decrease the recurring rate of periodontitis by cleaning the plaque left on the root surface. Periodontal pathogen can enter into blood stream through the ulceration epithelial resulting in bacteraemia, which may contribute to atheroma and atherosclerosis diseases. Atherosclerosis cardiovascular diseases are the main causes of death in the Western world [1]. The clinical and epidemiological findings confirmed an intimately association between atherosclerotic diseases and periodontitis. Prevalence and incidence of atherosclerotic diseases are significantly increased in periodontal infection patients [2]. The evidence has proved that severe periodontitis plays a role in the initiation and development of atherosclerosis disease [3]. Researcher has showed poor periodontal status is an important risk factor for cardiovascular diseases and the consistent relationship between pocket depth and incident myocardial infarction [4]. Periodontal bleeding per se is the indicator of bacteraemia and gingival inflammation is correlated positively with the prevalence of bacteraemia [5]. A meta-analysis suggested that individuals with periodontitis have 1.14 - 2.2 times greater risk of cardiovascular diseases compared to individuals without periodontitis [6]. In addition, experi- 
mental evidence has showed that periodontal pathogenic bacteria (Porphyromonas gingivalis) play a role in atherogenesis [7].

Increased inflammatory cytokines and C-reactive protein (CRP) levels may contribute to atherosclerosis diseases and periodontitis. Nonsurgical periodontal intervention may reduce atherosclerosis by inflammatory response regulating of cytokine and CRP levels. Periodontal therapy had effects on the modification of inflammatory indicators in systemically healthy people [8].

Whether severe periodontitis may lead to bacteraemia resulting in atherosclerosis diseases don't understand. The detailed mechanism of periodontal intervention on atherosclerosis diseases is still unclear. Whether periodontal intervention can be used as a therapeutic strategy to reduce atherosclerosis diseases is unknown. This study focuses on the influence of periodontitis and nonsurgical periodontal intervention on atherosclerosis diseases and prevents the morbidity and mortality of atherosclerosis cardiovascular disease.

\section{PERIODONTITIS AND PERIODONTAL INTERVENTION ON SYSTEMATIC INFLAMMATION}

Bacteria secretion and the accumulation of inflammatory mediators such as prostaglandin $E_{2}$ are the major causation factor leading to gingivitis, periodontal attachment loss and alveolar bone loss. The mechanism of prostaglandin $E_{2}$ on periodontitis is related to induce the blood vessel dilatation resulting in inflammatory cell and serum entering into the inflammatory tissue $[9,10]$. The experiments in animals and humans have showed that the Non-Steroidal Anti-Inflammatory Drugs (NSAID), especially indomethacin, had better therapeutic effects on gingivitis [11]. NSAID can control periodontitis signifycantly and inhibit periodontal supportive tissue destructtion in subjects with naturally occurring and animal experimental periodontitis [12]. Indomethacin can inhibit the inflammatory cytokine prostaglandin $\mathrm{E}_{2}$ formation in gingival and alveolar bone of guinea pigs to reduce the inflammatory response [13]. Inflammatory mediators of prostaglandin $E_{2}$ have been used as markers for active periodontal tissue breakdown and alveolar bone absorption many years ago. But the systemic effects of these local inflammatory markers have been largely neglected in 20 years ago. Recently, it has been showed that periodontal pathogens can invade gingival and periodontal tissues resulting in severe systemic infections such as atherosclerosis infections, brain abscesses [14], and pulmonary infections [15]. Such inflammation occurs as a result of periodontal pathogens in locations remote from the oral cavity.

Elevated interleukin 6 (IL-6) levels by bacterial lipopolysaccharide stimulated can promote coagulation and result in the development of atherosclerosis. Increased level of IL-6 is associated with both atherosclerosis and periodontitis. High plasma levels of tumor necrosis factor $\alpha$ (TNF- $\alpha$ ) and IL-6 have been associated with an increased risk of developing cardiovascular morbidity and mortality [16]. Peripheral blood mononuclear cells from periodontitis subjects released higher levels of TNF- $\alpha$ and IL-6 than those from healthy subjects. A prospective study of healthy men showed that the IL-6 levels of those who subsequently had a myocardial infarction were higher than control without myocardial infarction during a 6-year follow-up [17]. The results showed that IL-6 levels may be a predictor of risk of myocardial infarction in healthy men. Severe periodontitis can result in systemic inflammation characterized by elevated serum IL-6 [18]. A study showed that subjects with both coronary artery disease and periodontitis had significantly higher serum IL-6 compared with subjects with coronary artery disease who had no periodontitis [19]. TNF- $\alpha$ is related to the initiation and development of coronary artery and cardiovascular disease, and its levels increased in patients with periodontitis [20].

Periodontal intervention on periodontitis can reduce the levels of pro-inflammatory cytokines at three months post-therapy, and thus may lower the incidence of atherosclerotic diseases. In addition, the study showed that periodontal therapy had small impact on levels of TNF- $\alpha$ and IL-1 $\beta$ [21]. One study showed that periodontal therapy can lead to a decrease in IL-6 level [22]. In a recent study on coronary artery disease patients with periodontitis, periodontal therapy reduced serum IL-6 at 6 months post therapy [23]. Similar findings can be obtained in which periodontitis patients with coronary heart disease received periodontal therapy and control patients did not receive periodontal therapy. The serum IL-6 levels in periodontal therapy group decreased significantly than those in the control group at 3 months after periodontal therapy. A study reported serum TNF- $\alpha$ levels significant decreases after periodontal therapy [24]. Periodontal therapy is more positively related with the decrease of systemic markers of inflammation. Another periodontal therapy combined application of oral hygiene instructions with extraction of hopeless teeth, scaling and root planning and local controlled delivery of minocycline at all pockets $4 \mathrm{~mm}$ or deeper led to better improvements as compared with mechanical debridement alone [25] .

\section{PERIODONTITIS AND PERIODONTAL INTERVENTION ON PERIODONTAL PATHOGEN}

Periodontal bacteria mainly Gram-negative bacteria, which may secrete much of virulence factor act on host cells, can induce production inflammatory mediator on gingival and ligament and alveolar bone. The duration 
effects of bacteria and secretion on periodontal tissue resulted in periodontitis. Calculus which calcified by periodontal bacteria plaque may cause the ulceration of pocket epithelial. Periodontal pathogen in periodontal pocket can enter into blood through the ulcerated epithetlial leading to bacteraemia when periodontitis is severe. The transitory bacteraemia may not harm the body seriously, but the persistent and abundant bacteria to get together into the blood to stimulate the vessel epithelial may contribute to endocarditis. The congregated periodontal bacteria may contribute to the atheroma and atherosclerotic diseases. Acute gingivitis and poor periodontal status is correlated positively with the prevalence of bacteraemia. Bacteraemia induce the micro vascular thrombus and endangeitis, and farther to result in atherosclerotic diseases and acute myocardial infarction. Periodontal bacteria and bacterial products entering into the blood stream are thought to be one of the key initiators of biological events linking to atherosclerotic vascular diseases [26]. Periodontal pathogens are present in atherosclerotic plaques and may play a role in the development of atherosclerotic diseases. Increasing risk for systemic disease in subjects suffering from periodontal disease could be an increased prevalence and severity because of bacteraemia associated with periodontal bacteria [27]. In vitro study support that Porphyromonas gingivalis, as a model pathogen, may activate host immune responses associated with atherosclerosis. An in vivo study demonstrates that the bacteria can accelerate atheroma deposition in animal models [28]. Periodontal bacteria are associated with the infections at distant organs. In order to evaluate whether periodontal bacteria can detect from atherothrombotic plaques and blood vessel specimens, the bacterial DNA from periodontal pathogens has been analyzed in human arterectomy biopsies [29]. Another study suggested low concentrations of lipopolysaccharide from Porphyromonas gingivalis and Prevotella intermedia can stimulate the secretion of interleukins by human macrophages. Periodontal pathogens may increase the risk of atherosclerotic diseases through the platelet activation and aggregation [30].

About eight percent infectious can lead to endocarditic, which is more related to artery cirrhosis and acute myocardial infarction. The endocarditis is associated with perio-dontal diseases, including severe periodontitis and periodontal abscess. Therefore, study the strategy to interfere periodontitis is very important to prevent atherosclerosis cardiovascular diseases. Chen TL investigated the effects of periodontal scaling on bacteria in periodontal pocket and gingival crevicular fluid of the patients with periodontitis. Sixty cases with periodontitis were enrolled in the study and divided into periodontal scaling group and control group. The results showed that the percentage of bacillus, spirochetes of periodontal therapy group decreased more significantly and percentage of coccoid cells in pocket bottom increased more than control group. The gingival index, pocket depth and gingival crevicular fluid value were also decreased. The results represented that periodontal scaling can decrease the percentage of pathogenic bacteria in pocket bottom and decrease gingival index, pocket depth, and gingival crevicular fluid value [31].

\section{PERIODONTITIS AND PERIODONTAL INTERVENTION ON C-REACTIVE PROTEIN}

C-response protein (CRP) is an acute phase reactant protein and responsible for the increase in the vascular inflammation. The main function is thought to active the complement system. The CRP levels in human's plasma go up rapidly after an acute inflammatory response. Patients with periodontitis had elevated CRP levels. CRP is mainly synthesized by hepatocytes, which are stimulated by different cytokines, especially IL-6 [32]. CRP measurement can provide a more sensitive means of detecting signs of inflammation. CRP in serum is significantly increased in patients with coronary heart disease and myocardial infarction. Plasma CRP increased has been proved as a major risk factor for cardiovascular disease, and may be used as an important indicator of coronary artery disease and acute myocardial infarction. In a cohort study noted that increasing levels of serum CRP were associated with the risk of cardiovascular events, and that CRP was the strongest indication of such risk events [33]. CRP can increase low density lipoprotein uptake into macrophages and inhibit endothelial nitricoxide syntheses expression in aortic endothelial cells. CRP can also induce adhesion molecule expression in human endothelial cells in serum and may contribute to the hypercoagulative state in cardiovascular disease. Recently, the evidence has demonstrated the serum CRP increased in systemically healthy subjects with periodontitis [34]. Patients with periodontitis have higher circulating numbers of granulocytes and higher CRP concentrations when compared with periodontal healthy subjects.

A series of intervention trials have assessed the association between periodontitis and systemic inflammation. A study showed that periodontal therapy, which is composed of oral hygiene instructions, scaling and root planning and extraction of hopeless teeth, can result in a dose-dependent improvement in serum IL-6 and CRP. It was reported that subjects with periodontitis had 1.65 $\mathrm{mg} / \mathrm{l}$ higher serum CRP compared with individuals without periodontitis [35]. One study showed that periodontitis patients with cardiovascular diseases or hypertension had significantly higher serum CRP concentrations than patients without periodontitis [36]. Recently, clinical trial showed a significant reduction of CPR levels and in- 
flammatory markers in serum of the patients with periodontal diseases after periodontal therapy [37]. Periodontal intervention and maintenance may contribute to the reduction of systemic inflammation and the incidence and morbidity of cardiovascular diseases [38]. Therefore oral disinfection agent suggests to be used as a short-term mean to inhibit periodontal pathogens within $24 \mathrm{~h}$ [39].

\section{PERIODONTITIS AND PERIODONTAL INTERVENTION ON ENDOTHELIAL FUNCTION}

The bacteria and its virulence can change the vessel endothelium function and promote the congregate of monocyte and blood platelet. The denaturalization and collection of blood platelet can stimulate the blood vessel and induce the little blood vessel contracture. Periodontal pathogens and secretion may spread out through the blood stream and infect atherosclerotic plaques, leading to inflammatory response and destruction of the vessel epithelial function [40]. Periodontitis and systemic inflammation may impair vascular function. A study indicated that the patients with periodontitis had higher levels of endothelial dysfunction measured as flow-mediated dilatation of the brachial artery than control group [41]. Endothelial dysfunction is thought to be the first inflammatory response change of the blood vascular endothelial leading to arteriosclerosis. Severe periodontitis is associated with an increased risk of arteriosclerosis disease. Although the mechanism responsible for the relationship between periodontitis and arteriosclerosis diseases is not fully understood, chronic inflammatory periodontal diseases may lead to dysfunction of the vascular endothelium.

One study investigated the effects of periodontal therapy on vascular endothelial function. Twenty-two otherwise healthy subjects with moderate to severe periodontitis were enrolled and determined whether periodontal therapy would interfere endothelial function and inflammatory markers. The flow-mediated (endothelium dependent), nitroglycerin-mediated (endothelium-independent) dilation of the brachial artery, total and high-density lipoprotein cholesterol levels, and CRP and IL-6 in serum on baseline and 1 month after therapy were determined. The results showed that the periodontal therapy may improve periodontal pocket, flow-mediated dilation, and IL-6 in serum, as well as a trend to reduction CRP in serum, but there were no significant changes in nitroglycerin mediated dilation or in cholesterol levels. The study represented that improvement of endothelial function may be possible through elimination of severe periodontitis, and periodontal therapy may decrease inflammatory markers and improve the function of vascular endothelial [42]. Tonetti selected 120 patients with severe periodontitis and divided into periodontal care group (59 patients) or periodontal treatment group (61 patients). Endothelial function is assessed by measurement of the diameter of the brachial artery during flowmediated dilatation, and inflammatory markers and endothelial activation were evaluated before treatment and $1,7,30,60$, and 180 days after treatment. The results showed that the flow-mediated dilatation was signifycantly lower in the periodontal therapy group than control group, and the levels of CRP, IL-6, E-selection were significantly higher at 24 hours post-therapy. However, periodontal therapy resulted in significantly improved flowed-mediated dilatation and the plasma levels of Eselection compare with control group at 60 - 180 days post-therapy. The results represented that periodontal health was associated with improvement of endothelial function [43]. Seinost investigated the endothelial function of 30 patients with severe periodontitis and 31 periodontal healthy subjects. The flow-mediated dilatetion was significantly lower in patients with periodontitis than in control group before periodontal therapy. Periodontal therapy (including scaling and root planning, topical antimicrobials) exhibited concomitant improvement in flow-mediated dilatation of the brachial artery and CRP levels in serum [44].

\section{PERIODONTITIS AND PERIODONTAL INTERVENTION ON MICROCIRCULATION}

Inflammatory response of periodontal tissue can result in elevated lymphocyte and macrophage in gingival and periodontal ligament to interfere normal gingival and periodontal ligament microcirculation. Fifty-four patients with periodontitis and twenty-four periodontal health cases were enrolled in the experiment. The gingival blood flow, blood current velocity and blood concentration in gingival were measured by Laser Doppler Flowmeter, and the clinical periodontal indices were evaluated. The study showed that lower levels of gingival blood flow and blood current velocity were found in the periodontitis group than in the control group, and the higher levels of blood concentration was found in the periodontitis group than in the control group. The gingival blood flow, blood current velocity and blood concentration were more related to clinical indices and severity of periodontitis. Periodontitis can interfere with the gingival microcirculation [45].

A recent definitive trial investigated the effect of periodontal therapy (periodontal scaling and root planning) on the gingival microcirculation over 1 month period observation. The results showed that the gingival blood flow and blood velocity in gingival of aggressive and chronic periodontitis were increased at 1 month after 
periodontal therapy than therapeutic before. The gingival index and pocket depth at 1 month after periodontal therapy were improved than pre-therapy. The attachment loss of chronic periodontitis after periodontal therapy decreased than that of pre-therapy. The results showed that periodontal intervention had an important role in improving the gingival microcirculation, and the effect was associated with improving microcirculation [46]. Another study investigated the effects of periodontal scaling on gingival blood flow and gingivitis. The results showed that the gingival blood flow was increased significantly at 1 week after periodontal scaling, whereas the levels of gingival index, plaque index and calculus index decreased significantly after periodontal scaling. It showed that periodontal therapy could improve gingival microcirculation and alleviate gingivitis and periodontitis in marines [47].

\section{CONCLUSION}

Periodontitis is considered to be a potential risk factor for atheroma and atherosclerosis diseases. The durative severe periodontitis may increase bacteraemia odds. Periodontal inflammatory response could exacerbate vascular inflammation via secreted cytokines and pathogens that ultimately modulate atherosclerosis. The inflammatory cytokine, CRP levels increased, endothelial dysfunction, and periodontal bacteria reproduce and gingival microcirculation changed is association with atherosclerosis diseases. Nonsurgical periodontal intervention may prevent bacteremia resulting in atheroma and atherosclerosis by regulating inflammatory cytokine and CRP levels, and inhibiting periodontal pathogen, and improving the endothelial function and gingival microcirculation to reduce periodontitis, and decrease the incidence and morbidity of atherosclerosis cardiovascular diseases. Carefully designed randomized trials based on longer followup observation and the detailed mechanism is required to be investigated. It could be speculated that nonsurgical periodontal intervention may be used as the therapeutic strategy to interfere atherosclerosis diseases worldwide.

\section{ACKNOWLEDGEMENTS}

This study was supported by the Key Laboratory for Medicine of CPLA Foundation (036), and Naval Science \& Technology Program (2000-3327) and National Nature Science Foundation of China (3870831). We also wish to thank all the contributors for the substantial information that was compiled in previously published reviews that were cited in this manuscript. Their information was immensely helpful for preparing this manuscript.

\section{REFERENCES}

[1] Mattila, K.J., Asikainen, S., Wolf, J., Jousimies-Somer, H.,
Valtonen, V. and Nieminen, M. (2000) Age, dental infections, and coronary heart disease. Journal of Dental Research, 79, 756-760. doi:10.1177/00220345000790020901

[2] Ouyang, X.Y., Xiao, W.M., Chu, Y. and Zhou, S.Y. (2011) Influence of periodontal intervention therapy on risk of cardiovascular disease. Periodontology, 56, 227-257. doi:10.1111/j.1600-0757.2010.00368.x

[3] Geerts, S.O., Legrand, V., Charpentier, J., Albert, A. and Rompen, E.H. (2004) Further evidence of the association between periodontal conditions and coronary artery disease. Journal of Periodontology, 75, 1274-1280. doi:10.1902/jop.2004.75.9.1274

[4] Andriankaja, O.M., Genco, R.J., Dorn, J., Dmochowski, J., Hovey, K., Falkner, K.L. and Trevisan, M. (2007) Periodontal disease and risk of myocardial infarction: The role of gender and smoking. European Journal of Epidemiology, 22, 699-705. doi:10.1007/s10654-007-9166-6

[5] Guntheroth, W.G. (1984) How important are dental procedures as a cause of infective endocarditis? American Journal of Cardiology, 54, 797-801. doi:10.1016/S0002-9149(84)80211-8

[6] Bahekar, A.A., Singh, S., Saha, S., Molnar, J. and Arora, R. (2007) The prevalence and incidence of coronary heart disease is significantly increased in periodontitis: A metaanalysis. American Heart Journal, 154, 830-837. doi:10.1016/j.ahj.2007.06.037

[7] Brodala, N.M.E., Bellinger, D.A., Damrongsri, D., Offenbacher, S., Beck, J., Madianos, P., Sotres, D., Chang, Y.L., Koch, G. and Nichols, T.C. (2005) Porphyromonas gingivalis bacteremia induces coronary and aortic atherosclerosis in nor mocholesterolemic and hypercholesterolemic pigs. Arteriosclerosis, Thrombosis, and Vascular Biology, 25, 1446-1451. doi:10.1161/01.ATV.0000167525.69400.9c

[8] Ioannidou, E., Malekzadeh, T. and Dongari-Bagtzoglou, A. (2006) Effect of periodontal treatment on serum C-reactive protein levels: A systematic review and metaanalysis. Journal of Periodontology, 77, 1635-1642. doi:10.1902/jop.2006.050443

[9] Chen, T.L., Zhou, Y.J., Wu, Z.F., Yang, L.J. and Jin, Y. (1993) The distribution of prostaglandin $E_{2}$ in gingival of periodontal diseases. Journal of Practical Stomatology, 9, 73-75.

[10] Chen, T.L., Zhou, Y.J., Wu, Z.F. and Jin, Y. (1995) Quantitative analysis of prostaglandin $E_{2}$ in gingival of patients with periodontal diseases by radioimmunoassay and immunohistochemistry. Chin J Conserv Dent, 5, 141-143

[11] Chen, T.L. and Song, P.Z. (1996) The clinical observation of the effects of indomethacin on gingivitis in the seamen. Chinese Journal of Natural Medicines, 3, 104-106.

[12] Chen, T.L., Zhou, Y.J., Wu, Z.F., Song, P.Z. and Xu, Z.C. (1996) The inhibit effects of indomethacin on experimental periodontitis of guinea pigs, morphological observation. Journal of Stomatology, 16, 9-11.

[13] Chen, T.L., Zhou, Y.J. and Wu, Z.F. (1996) The inhibit effects of indomethacin on prostaglandin $\mathrm{E}_{2}$ in gingival and alveolar bone. Journal of Modern Stomatology, 10, 75-77. 
[14] Ewald, C., Kuhn, S. and Kalff, R. (2006) Pyogenic infections of the central nervous system secondary to dental affections-A report of six cases. Neurosurgery Reviews, 29, 163-166; Discussion 166-167. doi:10.1007/s10143-005-0009-1

[15] Suzuki, J.B. and Delisle, A.L. (1984) Pulmonary actinomycosis of periodontal origin. Journal of Periodontology, 55, 581-584. doi:10.1902/jop.1984.55.10.581

[16] Abeywardena, M.Y., Leifert, W.R., Warnes, K.E., Varghese, J.N. and Head, R.J. (2009) Cardiovascular biology of interleukin-6. Current Pharmaceutical Design, 15, 1809-1821. doi:10.2174/138161209788186290

[17] Ridker, P.M., Rifai, N., Stampfer, M.J. and Hennekens, C.H. (2000) Plasma concentration of interleukin-6 and the risk of future myocardial infarction among apparently healthy men. Circulation, 101, 1767-1772. doi:10.1161/01.CIR.101.15.1767

[18] Loos, B.G., Craandijk, J., Hoek, F.J., Wertheim-van D.P.M. and van der Velden, U. (2000) Elevation of systemic markers related to cardiovascular diseases in the peripheral blood of periodontitis patients. Journal of $\mathrm{Pe}$ riodontology, 71, 1528-1534. doi:10.1902/jop.2000.71.10.1528

[19] Higashi, Y., Goto, C., Hidaka, T., Soga, J., Nakamura, S., Fujii, Y., Hata, T., Idei, N., Fujimura, N., Chayama, K., Kihara, Y. and Taguchi, A. (2009) Oral infection- inflammatory pathway, periodontitis, is a risk factor for endothelial dysfunction in patients with coronary artery disease. Atherosclerosis, 206, 604-610. doi:10.1016/j.atherosclerosis.2009.03.037

[20] Czerniuk, M.R., Gorska, R., Filipiak, K.J. and Opolski, G. (2004) Inflammatory response to acute coronary syndrome in patients with coexistent periodontal disease. Journal of Periodontology, 75, 1020-1026. doi:10.1902/jop.2004.75.7.1020

[21] Buhlin, K., Hultin, M. and Norderyd, O. (2009) Periodontal treatment influences risk markers for atherosclerosis in patients with severe periodontitis. Atherosclerosis, 206, 518-522. doi:10.1016/j.atherosclerosis.2009.03.035

[22] Yamazaki, K., Honda, T., Oda, T., Ueki-Maruyama, K., Nakajima, T., Yoshie, H. and Seymour, G.J. (2005) Effect of periodontal treatment on the C-reactive protein and proinflammatory cytokine levels in Japanese periodontitis patients. Journal of Periodontal Research, 40, 53-58. doi:10.1111/j.1600-0765.2004.00772.x

[23] Higashi, Y., Goto, C., Hidaka, T., Soga, J., Nakamura, S., Fujii, Y., Hata, T., Idei, N., Fujimura, N., Chayama, K., Kihara, Y. and Taguchi, A. (2009) Oral infection-inflammatory pathway, periodontitis, is a risk factor for endothelial dysfunction in patients with coronary artery disease. Atherosclerosis, 206, 604-610. doi:10.1016/j.atherosclerosis.2009.03.037

[24] Iwamoto, Y., Nishimura, F., Soga, Y., Takeuchi, K., Kurihara, M., Takashiba, S. and Murayama, Y. (2003) Antimicrobial periodontal treatment decreases serum C-reactive protein, tumor necrosis factor-alpha, but not adiponection levels in patients with chronic periodontitis. Journal of Periodontology, 74, 1231-1236. doi:10.1902/jop.2003.74.8.1231
[25] D’Aiuto, F., Parkar, M., Nibali, L., Suvan, J., Lessem, J. and Tonetti, M.S. (2006) Periodontal infections cause changes in traditional and novel cardiovascular risk factors: Results from a randomized controlled clinical trial. American Heart Journal, 151, 977-984. doi:10.1016/j.ahj.2005.06.018

[26] Iwai, T. (2009) Periodontal bacteremia and various vascular diseases. Journal of Periodontal Research, 44, 689694. doi:10.1111/j.1600-0765.2008.01165.x

[27] Haraszthy, V.I., Zambon, J.J., Trevisan, M., Zeid, M. and Genco, R.J. (2000) Identification of periodontal pathogens in atheromatous plaques. Journal of Periodontology, 71, 1554-1560. doi:10.1902/jop.2000.71.10.1554

[28] Gibson, F.C., Yumoto, H. and Takahashi, Y. (2006) Innate immune signaling and Porphyromonas gingivalis-accelerated atherosclerosis. Journal of Dental Research, 85, 106-121. doi:10.1177/154405910608500202

[29] Gaetti-Jardim, E., Marcelino, S.L., Feitosa, A.C.R., Romito, G.A. and Avila-Campos, M.J. (2009) Quantitative detection of periodontopathic bacteria in atherosclerotic plaques from coronary arteries. Journal of Medical Microbiology, 58, 1568-1575.

doi:10.1099/jmm.0.013383-0

[30] Mattila, K., Nieminen, M., Valtonen, V., Rasi, V., Kesaniemi, Y., Syrjala, S., Jungul, P., Isoluoma, M., Hietaniemi, K., Jokinen, M. and Huttunen, J. (1989) Association between dental health and acute myocardial infarction. British Medical Journal, 298, 779-782. doi:10.1136/bmj.298.6676.779

[31] Chen, T.L., Lin, S.L., Zhou, Y.J., Yao, X.G., Liu, J.C.,Wu, Z.F. and Wang, G. (2006) The effects of periodontal scaling on microorganism in periodontal pocket and gingival crevicular fluid in patients with periodontitis. Journal of Practical Stomatology, 22, 547-549.

[32] Black, S., Kushner, I. and Samols, D. (2004) C-reactive protein. Journal of Biological Chemistry, 279, 4848748490. doi:10.1074/jbc.R400025200

[33] Ridker, P.M., Hennekens, C.H., Buring, J.E. and Rifai, N. (2000) C-reactive protein and other markers of inflammation in the prediction of cardiovascular disease in women. New England Journal of Medicine, 342, 836-843. doi:10.1056/NEJM200003233421202

[34] Sun, X.J., Meng, H.X., Shi, D., Xu, L., Zhang, L., Chen, Z.B., Feng, X.H., Lu, R.F. and Ren, X.Y. (2009) Elevation of C-reactive protein and interleukin-6 in plasma of patients with aggressive periodontitis. Journal of Periodontal Research, 44, 311-316. doi:10.1111/j.1600-0765.2008.01131.x

[35] Paraskevas, S., Huizinga, J.D. and Loos, B.G. (2008) A systematic review and meta-analyses on C-reactive protein in relation to periodontitis. Journal of Clinical Periodontology, 35, 277-290. doi:10.1111/j.1600-051X.2007.01173.X

[36] Tuter. G., Kurtis, B. and Serdar, M. (2007) Evaluation of gingival crevicular fluid and serum levels of high- sensitivity C-reactive protein in chronic periodontitis patients with or without coronary artery disease. Journal of $\mathrm{Pe}$ riodontology, 78, 2319-2324. doi:10.1902/jop.2007.070150 
[37] Offenbacher, S., Beck, J.D. and Moss, K. (2009) Results from the periodontitis and vascular events (PAVE) study: A pilot multicentered, randomized, controlled trial to study effects of periodontal therapy in a secondary prevention model of cardiovascular disease. Journal of $\mathrm{Pe}$ riodontology, 80, 190-201. doi:10.1902/jop.2009.080007

[38] Acharya, A., Bhavsar, N., Jadav, B. and Parikh, H. (2010) Cardioprotective: Effect of periodontal therapy in metabolic syndrome: A pilot study in Indian subjects. Metabolic Syndrome and Related Disorders, 8, 1-7. doi:10.1089/met.2010.0002

[39] Izumi, Y., Nagasawa, T. and Umeda, M. (2009) Periodontitis and cardiovascular diseases: The link and relevant mechanisms. Japanese Dental Science Review, 45, 98108. doi:10.1016/j.jdsr.2009.06.001

[40] Herzberg, M.C. and Weyer, M.W. (1998) Dental plaque, platelets, and cardiovascular diseases. Annals of Periodontology, 3, 151-160. doi:10.1902/annals.1998.3.1.151

[41] Amar, S., Gokce, N., Morgan, S., Loukideli, M., Van Dyke, T.E. and Vita, J.A. (2003) Periodontal disease is associated with brachial artery endothelial dysfunction and systemic inflammation. Arteriosclerosis Thrombosis Vascular Biology, 23, 1245-1249. doi:10.1161/01.ATV.0000078603.90302.4A

[42] Elter, J.R., Hinderlitwer, A.L., Offenbacher, S.,Beck, J.D., Caughey, M., Brodala, N. and Madianos, P.N. (2006) The effects of periodontal therapy on vascular endothelial function: A pilot trial. American Heart Journal, 151, 47.e1-47.e6.

[43] Tonetti, M.S., D Aiuto, F., Nibali, L., Danold, A., Storry, C., Parkar, M., Suvan, J., Hingorani, A.D., Vallance, P. and Deanfield, J. (2007) Treatment of periodontitis and endothelial function. New England Journal of Medicine, 356, 911-920. doi:10.1056/NEJMoa063186

[44] Seinost, G., Winmmer, G.,Skerget, M., Thaller, E., Brodmann, M., Gasser, R., Bratschko, R.O. and Pilger, E. (2005) Periodontal treatment improves endothelial dysfunction in patients with severe peridontitis. American Heart Journal, 149, 1050-1054. doi:10.1016/j.ahj.2004.09.059

[45] Chen, T.L., Lin, S.L., Lu, Y.L., Liu, J.C. and Liu, G.Q. (1999) The blood flow, blood current velocity and blood concentration in gingival of the patients with periodontal diseases. Journal of Practical Stomatology, 15, 33-34.

[46] Chen, T.L., Lin, S.L., Zhou, Y.J., Liu, J.C. and Wu, Z.F. (2007) The biological effects of periodontal scaling and root planning on gingival microcirculation. Journal of Modern Stomatology, 21, 4-6.

[47] Chen, T.L., Lin, S.L., Liu, G.Q., Wang, W.X., Yu, J.W., Liu, J.C., Zhao, H.J. and Ji, Q.J. (2007) Effects of periodontal scaling on gingival blood flow volume and gingivitis in marines. Chinese Journal of Nautical Medicine and Hyperbaric Medicine, 14, 155-157. 